\title{
The role of chronic cerebrospinal venous insufficiency in multiple sclerosis
}

\author{
O papel da insuficiência venosa crônica cérebro-espinhal na esclerose múltipla
}

\author{
Arnold Radu ${ }^{1}$, Fabrício Guimarães Gonçalves ${ }^{2}$
}

Multiple sclerosis is a non-hereditary neurodegenerative disease caused by an autoimmune process that results in demyelination of axons in the central nervous system thus leading to a broad spectrum of neurological symptoms. The etiology of multiple sclerosis is multifactorial and a single cause explaining all aspects of the disease has not yet been identified. Authors have agreed that this disorder is most likely due to a combination of factors, which includes a genetic predisposition, combined with environmental elements and possibly an infectious component.

The cascade is initiated by the blood-brain barrier breakdown. This allows T-cell infiltration into the central nervous system, which triggers an inflammatory process that causes axonal myelin sheath damage resulting in poor neuronal action potential propagation. Oligodendrocytes attempt to remyelinate, although insufficient to compensate for demyelination in the long term. The end result is permanent neuronal scarring and damage and loss of parenchyma ${ }^{(1)}$.

According to the National Multiple Sclerosis Society, multiple sclerosis patients may present three distinct clinical courses: relapsing remitting, primary progressive, and secondary progressive ${ }^{(2)}$. In the last five years, additional propositions have been made to unveil the causes of multiple sclerosis. Zamboni has raised the possibility that multiple sclerosis and chronic venous disease may share some key features, particularly, increased iron deposition in the brain ${ }^{(3)}$. This has been first described by Adams et al.: "In active multiple

1. BSc, MD, Radiology Resident, McGill University Health Centre (MUHC), Montreal, Quebec, Canada.

2. MD, Neuroradiology Fellow, McGill University Health Centre (MUHC), Montreal, Quebec, Canada. E-mail: goncalves.neuroradio@ gmail.com sclerosis cases, hemosiderin is found in the areas of intense edema and myelin pallor, often associated with marked perivenular lymphocytic cuffing"(4). This hypothesis has been supported by the initial observation of an unusual reflux from the chest into the internal jugular veins of a multiple sclerosis patient during a Doppler examination of the carotid arteries during Valsalva maneuver. This abnormal blood flow pattern has been termed chronic cerebrospinal venous insufficiency and was subsequently observed in other multiple sclerosis patients. According to the Italian research team, this inadequate cerebral venous outflow would decrease shear stress in the vessels walls, increasing the transendothelial permeability and ultimately disrupting the blood-brain barrier, which would allow erythrocyte extravasation into the perivenous region. The macrophage degradation of extravasated red blood cells would create tissue iron overload and the resulting hemosiderin-laden macrophages would then release cytokines which activates T-lymphocyte recruitment and attack of the myelin sheath. Finally, the development of B-cells with antibodies against myelin would lead to a chronic autoimmune reaction.

The diagnosis of chronic cerebrospinal venous insufficiency can be performed with Doppler ultrasonography of the neck to evaluate the internal jugular and vertebral veins, or with transcranial color-coded Doppler sonography to evaluate the blood flow in the deep cerebral veins. Both examinations must be performed twice, once in the supine and subsequently in the sitting position.

In the case of Doppler ultrasonography of the neck, the aim is to identify the presence of reflux in the internal jugular veins and/or vertebral veins, reflux in any of the deep cerebral veins, evidences of internal jugular 
veins stenoses, flow not detectable by Doppler in the internal jugular veins and/or vertebral veins and reverted postural control of the main cerebral venous outflow pathways. Normally, in the supine position the outflow is predominant through the vertebral veins, while in the sitting position it should happen via the internal jugular veins. Reflux in the internal jugular veins or in the vertebral veins is considered present if there is a reversal of flow lasting more than $0.88 \mathrm{sec}-$ ond. In healthy patients, the venous flow is always directed towards the heart regardless of the position of the head.

With transcranial color-coded Doppler sonography, reflux in the deep cerebral veins is considered when it lasts more than half a second. Normally, the flow is unidirectional and away from brain in both supine and sitting positions. Chronic cerebrospinal venous insufficiency is considered to be present when two of these sonographic criteria are met, becoming also an indication for venography.

Zamboni et al. have performed venography in a heterogeneous group of 65 patients suffering from either of the three major types of multiple sclerosis and found them to be positive for chronic cerebrospinal venous insufficiency according to the aforementioned criteria $^{(5)}$. For cases in which the lumen reduction was greater than $50 \%$, angioplasty was performed exclusively at the levels of the internal jugular veins and/or azygos veins. The group concluded that endovascular treatment of chronic cerebrospinal venous insufficiency is feasible and safe with a negligible rate of complications. Endovascular treatment successfully decreased the venous pressure in all treated segments with statistical significance between pre- and postoperative values. The data showed that relapsing remitting patients significantly improved eighteen months post angioplasty, while primary progressive and secondary progressive patients had limited improvement at six months and no significant improvement after eighteen months. All the relapsing remitting patients with post-procedural patency of internal jugular and azygos veins and remained relapse-free through the whole study period, and the number of patients with active lesions at MRI decreased from $50 \%$ to $12 \%$.

According to Zamboni et al., clinically defined multiple sclerosis is strongly associated with chronic cerebrospinal venous insufficiency, somehow linked to abnormal venous hemodynamics caused by multiple extracranial venous strictures ${ }^{(6)}$. Overall, angioplasty seems to be a promising avenue mainly for the relapsing remitting multiple sclerosis course, offering significant improvement in long-term neurological outcome with no relapses in patients with stable venous patency. Patency rates could hypothetically be further improved by stent insertion; however there is currently low availability of such devices with appropriate size and length in the market.

\section{REFERENCES}

1. Compston A, Coles A. Multiple sclerosis. Lancet. 2002;359: 1221-31.

2. Lublin FD, Reingold SC. Defining the clinical course of multiple sclerosis: results of an international survey. National Multiple Sclerosis Society (USA) Advisory Committee on Clinical Trials of New Agents in Multiple Sclerosis. Neurology. 1996; 46:907-11.

3. Zamboni P. The big idea: iron-dependent inflammation in venous disease and proposed parallels in multiple sclerosis. J R Soc Med. 2006;99:589-93.

4. Adams CW. Perivascular iron deposition and other vascular damage in multiple sclerosis. J Neurol Neurosurg Psychiatry. 1988; 51:260-5.

5. Zamboni P, Galeotti R, Menegatti E, et al. A prospective openlabel study of endovascular treatment of chronic cerebrospinal venous insufficiency. J Vasc Surg. 2009;50:1348-58 e1-3.

6. Zamboni P, Galeotti R, Menegatti E, et al. Chronic cerebrospinal venous insufficiency in patients with multiple sclerosis. J Neurol Neurosurg Psychiatry. 2009;80:392-9. 